\section{Incidentaloma de Supra-Renal: Manejo Diagnóstico e Terapêutico}

\section{RESUMO}

Descrevemos o caso de paciente que apresentou massa em suprarenal direita na ultra-sonografia abdominal durante investigação de dor abdominal, caracterizando um incidentaloma de supra-renal; a tomografia computadorizada confirmou a presença de lesão. A avaliação endócrina detectou perda do ritmo circadiano do cortisol e sua não supressibilidade pela dexametasona, caracterizando síndrome de Cushing. Entretanto, a paciente não apresentava as características clínicas da síndrome, o que sugeriu o diagnóstico de síndrome de Cushing subclínica. A paciente fol submetida a cirurgia laparoscópica para ressecção da glândula adrenal direita cujo diagnóstico anátomo-patológico foi de adenoma de supra-renal. Uma nova avaliação do cortisol foi feita após a cirurgia com resultados normais. Pretendemos sugerir neste relato um manejo racional e com baixos custos, dos incidentalomas de supra-renal. (Arq Bras Endocrinol Metab 2000; 44/6: 523-7)

Unitermos: Incidentaloma de supra-renal; Adenoma adrenal; Sindrome de Cushing: Sindrome de Cushing subclínica.

\begin{abstract}
We report a patient who presented a mass in the right adrenal gland during ultra-sound imaging for the investigation of abdominal pain, characterizing an adrenal incidentaloma; computed tomography confirmed the presence of the lesion. Endocrine evaluation detected loss of circadian rhythm of cortisol production and its non suppressibility to dexamethasone testing, characteristics of the presence of Cushing's syndrome. However, the patient did not present typical clinical manifestations, suggesting subclinical Cushing's syndrome. Laparoscopic surgery for ressection of the right adrenal gland was performed with a pathology diagnosis of adrenal adenoma. Normal cortisol levels were found on re-evaluation. We propose in this report a rational and inexpensive management for adrenal incidentalomas.
\end{abstract} (Arq Bras Endocrinol Metab 2000; 44/6: 523-7)

Keywords: Adrenal incidentaloma; Adrenal adenoma; Cushing's syndrome; Subclinical Cushing's syndrome.

\section{APRESENTAÇĀO DO CASO}

$\mathrm{U}$ MA SENHORA DE 51 anOS consultou em Posto de Saúde periférico com queixa de dor em hipocôndrio direito há aproximadamente um mês, contínua, diária, de moderada intensidade, sem irradiação, sem fatores associados de alívio ou piora. Uma ultra-sonografia abdominal total, solicitada por suspeita de colelitíase, mostrou uma massa em topografia de glândula suprarenal direita medindo $4,5 \mathrm{~cm}$ em seu maior diâmetro, sendo a paciente então

\section{apresentação de caso}

\author{
Alessandra T. de Oliveira \\ Vera M.F. Silveira \\ Ary Carnieletto Jr \\ Eduardo M. Rotta \\ Róger R. Berçot
}

\author{
Serviço de Clínica Médica, \\ Hospital da Fundação de \\ Apoio Universitário (FAU), \\ Faculdade de Medicina da \\ Universidade Federal de Pelotas, \\ Pelotas, RS.
}




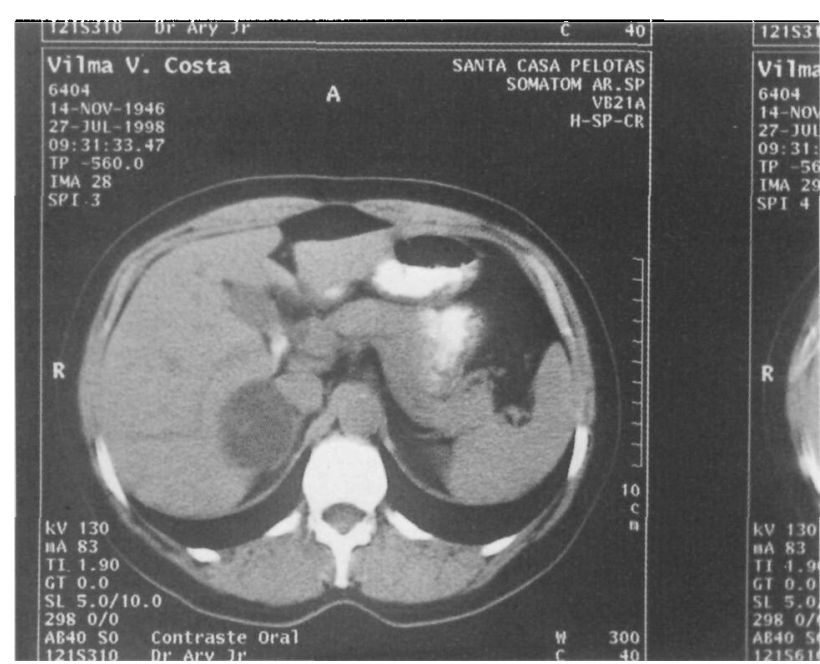

Figura 1. Corte tomográfico da lesão em supra-renal direita.

encaminhada para o Serviço de Clínica Médica do Hospital da FAU para investigação.

Durante a avaliação clínica a paciente negou sintomas atuais ou condições prévias e não apresentou características de hiper ou de hipofunção supra-renal ou alterações em qualquer aparelho ou sistema ao exame fisico.

Realizou-se investigação para metástases $\mathrm{em}$ supra-renal de tumor de mama, pulmão, rim, melanoma, gastrointestinal e linfoma, todos com resultados normais.

TC abdominal total com duplo contraste mostrou massa de contornos lisos e regulares de densidade mista, em topografia de supra-renal direita, medindo 5,0 x 4,0 cm em seus maiores eixos, sendo os demais aspectos normais (figura 1 ).

\section{Exames laboratoriais:}

Potássio: $4,7 \mathrm{mEq} / \mathrm{L}(3,5-5,0)$; testosterona livre: $0,65 \mathrm{ng} / \mathrm{dL}(0,3-1,9)$; S-DHEA: $11 \mathrm{mcg} / \mathrm{dL}(35-$ $400)$; androstenediona: $0,5 \mathrm{ng} / \mathrm{mL}(0,4-2,7)$.

Cortisol basal às $9: 00 \mathrm{~h}: 8,95 \mathrm{mcg} / \mathrm{dL}(4,3-22,4)$; às 17:00h: $25,8 \mathrm{mcg} / \mathrm{dL}(3,0-16,7)$

Cortisol após supressão com $\mathrm{lmg}$ de dexametasona: $12,4 \mathrm{mcg} / \mathrm{dL}$

Catecolaminas urinárias: epinefrina: $21,3 \mathrm{mcg} / 24 \mathrm{~h}$ $(<60)$; norepinefrina: $75,2 \mathrm{mcg} / 24 \mathrm{~h}(<200)$; dopamina: $122,7 \mathrm{mcg} / 24 \mathrm{~h}(65-400)$

Ácido Vanilil-Mandélico urinário: $5,4 \mathrm{mg} / 24 \mathrm{~h}(<12)$ Cortisol livre urinário (CLU) basal: $397,7 \mathrm{mcg} / 24 \mathrm{~h}$ $(28,5-214)$

CLU após supressão com 8mg de dexametasona: 214 $\mathrm{mcg} / 24 \mathrm{~h}$

A paciente foi submetida à videoendoscopia para adrenalectomia direita.
Anátomo-patológico: Macroscopia: vários fragmentos irregulares de tecido pesando em conjunto $60 \mathrm{~g}$, alaranjados, friáveis. Microscopia: presença de células claras.

Diagnóstico: adenoma de supra-renal.

Evolução clínica:

A paciente não apresentou complicações no pós operatório, recebendo alta hospitalar no segundo dia após a cirurgia.

Exames laboratoriais dois meses após a cirurgia: Cortisol basal às $9: 00 \mathrm{~h}: 15,9 \mathrm{mcg} / \mathrm{dL}(4,3-22,4)$; às 17:00h: $7,9 \mathrm{mcg} / \mathrm{dL}(3,0-16,7)$

Exames laboratoriais dois anos após a cirurgia: Cortisol basal às $9: 00 \mathrm{~h}: 14,8 \mathrm{mcg} / \mathrm{dL}(4,3-22,4)$; às $17: 00 \mathrm{~h}: 5,5 \mathrm{mcg} / \mathrm{dL}(3,0-16,7)$

\section{DISCUSSĀO}

A melhor resolução dos exames de imagem: tomografia computadorizada (TC), ressonância magnética e ultra-sonografia, têm aumentado a deteç̧ão incidental de tumores em supra-renal (1-3). Os incidentalomas de supra-renal são definidos como massas encontradas incidentalmente nessa glândula ao se realizar um exame de imagem do abdome a partir de outras indicações (1-6). As causas mais comuns são: dor lombar, hematúria, dor abdominal e nefrolitíase $(2,4,7)$.

A prevalência dos incidentalomas de supra-renal $\mathrm{em} \mathrm{TC}$ abdominal varia de 1 a $2 \%(1-5) \mathrm{e} \mathrm{cm} \mathrm{estudos}$ de necrópsia de 1,4 a $8,7 \%(1,3,5,8)$. A idade média dos pacientes com incidentaloma de supra-renal é de aproximadamente 56 anos $(4,9)$, a maioria do sexo feminino $(2,4,7,9)$.

Após a detecção da massa a conduta diagnóstica deve direcionar-se para identificar se existe um tumor primário ou secundário, presença de malignidade e secreção hormonal $(1,5,8,10)$.

Todos os pacientes devem sofrer investigação para os tumores que mais freqüentemente metastatizam para supra-renais $(1,4,7,8,10,11)$ como: mama, pulmão, melanoma, rim, estômago, coloretal e linfoma ou outros, de acordo com o quadro clínico.

Para o diagnóstico da lesão primária avaliam-se as características encontradas na TC: lesão pequena, bem delimitada, homogênea, sugere benignidade $(4,10)$. Já os critérios tomográficos de malignidade incluem: lesões grandes, heterogêneas, com margens pouco definidas e invasão local $(2,3,10)$, e extensão do tumor para veia cava inferior que pode aparecer após injeção de con- 
traste endovenoso (10). Contudo a TC não é segura para diferenciar lesões benignas de malignas.

A massa adrenal incidental mais freqüente é o adenoma não funcionante $(4,7,10,11)$. Entretanto, em todos os pacientes nos quais se detecta um incidentaloma de supra-renal deve ser feita avaliação laboratorial para identificar os principais tumores funcionantes, sobretudo porque mais de $50 \%$ dos carcinomas adrenocorticais são hormonalmente ativos $(2,5,11)$. Estudos relatam uma incidência anual de carcinoma adrenal entre $0,1 / 45.000$ a $0,01 / 17.000(1,2,4,9,11)$.

$\mathrm{Na}$ maior parte dos casos, tumores "clinicamente silenciosos" são formas sutis de síndrome de Cushing (12). O feocromocitoma, embora raro ( $1,5 \%$ a $13 \%)(12)$, deve ser investigado em todos os pacientes nos quais se diagnosticar uma massa incidental em supra-renal, já que é potencialmente letal $(1,2,5,10,12)$. Sua identificação no pré-operatório é extremamente importante. Kloos et al. (10) relatam várias séries mostrando que mais de $80 \%$ dos pacientes com feocromocitoma não diagnosticado morrem ao serem submetidos a cirurgia ou à anestesia. $\mathrm{Na}$ ausência de hipertensão arterial e outros sintomas existe um valor preditivo negativo de $99,6 \%$ para feocromocitoma $(1,5)$; mesmo assim, deve-se dosar o ácido vanililmandélico e/ou metanefrinas e catecolaminas urinárias (1-5,8-10,13-16).

A investigação do excesso de mineralocorticóides consiste em dosar o potássio sérico (1-5,8-10) e, principalmente, em aferir a pressão arterial, pois praticamente todos os pacientes com essa disfunção apresentam hipertensão arterial no momento do diagnóstico $(1,2,5,9)$. Na presença de hipocalemia deve-se medir ainda a atividade plasmática da renina (APR) $(4,5)$ e a concentração da aldosterona plasmática $(1,4,5)$. A APR baixa e alta concentração de aldosterona sugerem o diagnóstico de hiperaldosteronismo primário $(4,5)$. A freqüência dos aldosteronomas entre as massas incidentais adrenais chega a 7\% (10).

Os testes básicos para investigação do excesso de cortisol incluem $(2,3,5,7,9,10,13,15,17)$ : a dosagem do cortisol plasmático às $8 \mathrm{e}$ às 18 horas, o cortisol livre urinário e o teste de supressão com $1 \mathrm{mg}$ e com $8 \mathrm{mg}$ de dexametasona.

A síndrome de Cushing é detectada com a perda do ritmo diurno do cortisol (9) e com a ausência de supressão do cortisol pela dexametasona (5). Quando os achados laboratoriais não são acompanhados das características clínicas daquela doença, como no presente caso, tem-se a sindrome de Cushing subclínica $(1,2,5,10)$. Até o momento permanece incerto qual a melhor investigação e conduta frente a essas lesões, pois não se sabe quantas progredirão para a síndrome de Cushing clinicamente florida $(2,3,5,8,9)$.

O estudo dos hormônios sexuais nos incidentalomas de supra-renal consiste em dosar: testosterona, androstenediona e o sulfato de deidroepiandrosterona (S-DHEA) $(4,7,8,9,11)$. Alguns autores, entretanto, preconizam que somente o S-DHEA seja solicitado para todos os pacientes pois os adenomas secretores de hormônios feminilizantes ou masculinizantes são raros, de modo que os demais hormônios devem ser solicitados como screening em pacientes sintomáticos $(2,3)$. Níveis de S-DHEA abaixo do normal têm sido vistos em associação com hipersecreção autônoma de cortisol em adenomas de supra-renal. O ACTH plasmático é suprimido e, conseqüentemente, causa redução do S-DHEA. O carcinoma adrenocortical tende a apresentar níveis elevados de S-DHEA $(3,5,10)$.

Kloos et al (10) sugerem que os pacientes com exames funcionais normais sejam submetidos à cintilografia adrenal com NP59 (131 I- $\beta$ iodo-metilnorcolesterol) porque esta identifica de forma não invasiva e com boa acurácia os adenomas benignos não funcionantes. Lesōes malignas primárias de adrenal, metástases, lesões císticas e hematomas apresentam um padrão "discordante" à cintilografia, ou seja, a glândula contralateral à lesão ć a captante do contraste enquanto os adenomas adrenais são "concordantes": o acúmulo do NP 59 é ipsolateral à lesão $(1,5,10)$.

Gross et al. $(3,9,10)$ avaliando 229 pacientes com incidentalomas adrenais não funcionantes mostrou que a cintilografia tem uma sensibilidade de $71 \%$, especificidade de $100 \%$, acurácia de $93 \%$, valor preditivo negativo de $91 \%$ (lesões concordantes e não lateralizantes) e valor preditivo positivo de $100 \%$ (lesões discordantes) para diferenciar adenoma benigno não funcionante de carcinoma adrenocortical.

A punção aspirativa por agulha fina guiada por TC é outro método que pode ser usado para ajudar a diagnosticar a massa adrenal incidental. Porém esse procedimento não é isento de riscos, entre eles: pneumotórax, pancreatite, hemorragia e hipotensão $(1,5,10)$, de modo que só deve ser usado em casos de dúvida quanto ao tipo de massa (5) e quando seus resultados influenciarem na terapêutica do paciente (1). É eficaz para distinguir entre tecido adrenal $\mathrm{e}$ não adrenal $(1,2,5,9-11)$. Dificilmente difere lesões adrenais benignas de lesões malignas primárias bem diferenciadas, como na maioria dos tecidos endócrinos $(9,10)$, pois como no adenoma folicular da tireóide a diferença entre os adenomas benignos e os carcinomas bem diferenciados requer a cvidência, 
neste último, de invasão capsular ou vascular $(5,9)$. Silverman et al. alcançaram $86 \%$ de diagnóstico correto na biópsia inicial; Bernardino et al. $83 \%$, em quatro de suas noves falhas a punção foi repetida de modo que incluindo as duas tentativas a acurácia diagnóstica total atingiu 90,6\% $(1,10)$.

Muitas vezes a histologia não consegue diferenciar um adenoma de um carcinoma $(18,19)$, porém existem critérios diagnósticos de malignidade como: necrose, bandas fibróticas, invasão vascular, número de mitoses por campo de grande aumento (19). Há casos, também, em que só com o tempo poderá se ter o diagnóstico cxato da lesão pelo aparecimento de metástases à distância (19).

A ressecção cirúrgica dos tumores adrenais deve ser feita em todas as lesões funcionantes $(1-3,5,7-9,11) \mathrm{e}$ lesões não funcionantes com $4 \mathrm{~cm}$ ou mais, haja visto que quanto maior a lesão, maior é o risco de se ter presente uma lesão maligna $(3,7,8,10-13,15)$. Há autores que defendem a idéia de que lesões não funcionantes, que não preencham os critérios de malignidade para serem ressecados, necessitam seguimento clínico e radiológico (1-3,5,8-12). Maiores investigações podem ser obtidas com ressonância magnética, punção aspirativa por agulha fina c cintilografia $(1,9)$.

As técnicas cirúrgicas laparoscópicas têm aumentado de forma crescente sua importância desde 1992 na cirurgia da supra-renal (6), oferecendo certas vantagens sobre a cirurgia convencional, como menor agressividade, cicatrizes mais simples e indolores, tempo de hospitalização reduzido e retorno mais precoce às atividades profissionais (20). A laparoscopia tem sua indicação quando: não há suspeita de malignidade, a lesão é unilateral, paciente não tenha sido submetido a cirurgias abdominais prévias (18). Algumas vezes é necessário a conversão da cirurgia laparoscópica em convencional, como em casos $\mathrm{em}$ que se detectou malignidade no trans-operatório, lesões difíceis de dissecar ou ainda na ocorrência de hemorragia, complicação mais temida na laparoscopia (18). Inicialmente utilizava-se somente a via transperitoneal que apresentava uma pequena taxa de complicações (12\%) (6). Como a via anterior transperitoneal atravessa toda a cavidade peritoneal, há o risco de lesar órgãos intra abdominais, o que levou ao desenvolvimento da via retroperitoneoscópica, que tem a vantagem de poder ser utilizada em pacientes com cirurgias abdominais prévias (6).

No presente relato, mostramos a avaliação clínica, laboratorial e radiológica de uma paciente com uma massa incidental em supra-renal direita $\mathrm{e}$ a melhor abordagem cirúrgica neste caso.

\section{CONCLUSÃO}

No caso estudado voltamos nossa atenção para definir se a lesão encontrada era funcionante. A paciente não apresentava sinais de hirsutismo, encontrava-se normotensa, e o potássio sérico estava em níveis dentro da normalidade. Apesar de não ser constatado sinais ou sintomas de Síndrome de Cushing, os exames laboratoriais detectaram hipercortisolismo. Utilizamos como screening inicial para caracterizar o quadro como síndrome de Cushing subclínica a dosagem do cortisol sérico após supressão com lmg de dexametasona; como não houve supressão [cortisol $<5 \mathrm{mcg} / \mathrm{dL}$ (17)], um segundo teste e a dosagem de cortisol livre urinário (CLU), foram solicitados para confirmar o hipercortisolismo.

Como não dispunhamos do $\mathrm{CRH}$ para distinção etiológica da síndrome de Cushing, utilizamos o teste de supressão com altas doses de dexametasona (teste de Liddle 2, modificado) com dosagem do cortisol sérico ou urinário, como na nossa paciente, iniciando a coleta às $8 \mathrm{~h}$ da manhã seguinte à dose de $8 \mathrm{mg}$ de dexametasona ( $2 \mathrm{mg}$ de $6 / 6 \mathrm{~h}$ por $24 \mathrm{~h}$ ). A supressão $>50 \%$ do CLU é indicativo de doença de Cushing, enquanto que a supressão $<50 \%$, como no caso relatado, é sugestivo de tumor adrenal. Uma supressão $>\mathbf{5 0} \%$ também pode ser raramente observada em casos de tumor adrenal, bem como em $10-20 \%$ dos casos de secreção ectópica de ACTH (SEA). Não foi possível realizar à época a dosagem de ACTH plasmático, que geralmente encontra-se suprimido $(<5 \mathrm{pg} / \mathrm{mL})$ em tumores de supra-renal, normal ou elcvado na doença de Cushing e elevado na SEA.

Como em nosso hospital contamos com cirurgiòes experientes em videolaparoscopia, aliado as características da lesão da paciente, optou-se por este tipo de cirurgia pelas vantagens em relação à cirurgia convencional, confirmando os dados bibliográficos. Não houve complicações no pós operatório de modo que dois dias após o ato cirúrgico a paciente recebeu alta hospitalar voltando as suas atividades diárias usuais.

\section{AGRADECIMENTOS}

Agradecemos ao Dr. Vicente Petrollini Carvalho pela traduçào dos artigos em alemão que colaboraram na revisào deste trabalho.

\section{REFERÊNCIAS}

1. Li BDL, Douglass HO. Management of the incidentally discovered adrenal mass or "incidentaloma". J LA Stale Med Soc 1997;149:291-9. 
2. Bastounis EA, Karayiannakis AJ, Anapliotou MLG, Nakopoulou L, Makri GG. Papalambros EL. Incidentalomas of the adrenal gland: diagnostic and therapeutic implications. Am Surg 1997:63:356-60

3. Bondanelli M. Campo M, Trasforini $G$, Ambrosio MR, Zatelli MC. Franceschetti $P$, et al. Evaluation of hormonal function in a series of incidentally discovered adrenal masses. Metabolism 1997;46:107-13.

4. Barili CA, Czepielewski MA. Incidentalomas de suprarenal: Análise de uma casuística. Ara Bras Endocrinol Metab 1997;39:147-52.

5. Newell-Price J, Grossmam AB. Adrenal incidentaloma: subclinical Cushing's syndrome. Postgrad Med J 1996:72:207-10.

6. Heintz A, Beyer J, Junginger TH. Endoscopic surgical therapy of accidentally diagnosed tumors of the adrenal gland. Zentralblatt für Chirurgie 1997:122:477-80

7. Kasperlik-Zaluska AA, Roslonowska E, Slowinska-Srzednicka J. Migdalska B. Jeske W. Makowska A, et al. Incidentally discovered adrenal mass (incidentaloma): investigation and management of 208 patients. Clin Endocrinol 1997:46:29-37.

8. Bencsik ZS, Szabolcs I, Góth M, Vörös A, Kaszás I, Gönczi $J$, et al. Incidentally detected adrenal tumours (incidentalomas): histological heterogeneity and differentiated therapeutic approach. J Intern Med 1995:237:585-9.

9. Sirén JE, Haapiainen RK, Huikuri KT, Sivula AH, Incidentalomas of the adrenal gland: 36 operated patients and review of literature. World J Surg 1993:17:634-9.

10. Kloos RT, Gross MD, Francis IR, Korobkin M, Shapiro B. Incidentally discovered adrenal masses. Endocr Rev 1994; 16:460-84.

11. Hensen J, Harsch I, Sachse R, Pavel M. Rico AF, Walter P, et al. The adrenal incidentaloma is no time bomb - argu- ments for a follow up. Zentralblatt für Chirurgie $1997 ; 122: 487-93$

12. Mantero F, Arnaldi $G$. Investigation protocol: adrenal enlargement. Clin Endocrinol 1999:50:141-46.

13. Osella $G$, Terzolo $M$, Borretta $G$, Magro $G$ P. Ali $A$ Piovesan $A$, et al. Endocrine evaluation of incidentally discovered adrenal masses (Incidentalomas). J Clin Endocrinol Metab 1994:79:1532-39.

14. Gross M D, Shapiro B. Clinical Review 50. Clinically silent adrenal masses. J Clin Endocrinol Metab 1993;77:885-88.

15. Barzon L, Scaroni C, Sonino N, Fallo F, Gregianin M, Macri C, et al. J Clin Endocrinol Metab 1998:83:55-62.

16. Terzolo M, Osella G, Ali A, Borretta G, Magro GP, Termine $A$, et al. Different patterns of steroid secretion in patients with adrenal incidentaloma. J Clin Endocrinol Metab 1996;81:740-4.

17. Vilar L, Castelar E, Moura E, Leal E, Machado AC. Teixeira L, et al. Endocrinologia Clínica. São Paulo:Medsi, 1999

18. Cotran RS, Kumar $V$, Robbins SL. Patologia Estrutural e Funcional. Rio de Janeiro:Guanabara, 1991.

19. DeVita Jr VT, Hellmann S, Rosenberg SA. Cancer Principles e Practice of Oncology. New York: LippincottRaven, 1997

20. Henry JF, Deizot A, Puccini M, Ayari R. Role of laparoscopy approach to the adrenal glands. J Chirur 1996; $133: 111-6$.

\section{Endereço para correspondência:}

Alessandra Teixeira de Oliveira Rua Miguel Barcellos, 305 apto 403 96015-150 Pelotas, RS 